\title{
ON THE ABSOLUTE RUIN IN A MAP RISK MODEL WITH DEBIT INTEREST
}

\author{
ZHIMIN ZHANG, ${ }^{* * *}$ Chongqing University \\ HAILIANG YANG, ${ }^{* * *}$ The University of Hong Kong \\ HU YANG, ${ }^{*}$ Chongqing University
}

\begin{abstract}
In this paper we consider a risk model where claims arrive according to a Markovian arrival process (MAP). When the surplus becomes negative or the insurer is in deficit, the insurer could borrow money at a constant debit interest rate to repay the claims. We derive the integro-differential equations satisfied by the discounted penalty functions and discuss the solutions. A matrix renewal equation is obtained for the discounted penalty function provided that the initial surplus is nonnegative. Based on this matrix renewal equation, we present some asymptotic formulae for the discounted penalty functions when the claim size distributions are heavy tailed.
\end{abstract}

Keywords: MAP; absolute ruin; discounted penalty function; matrix renewal equation; asymptotic; heavy-tailed distribution

2010 Mathematics Subject Classification: Primary 91B30

Secondary 91B70

\section{Introduction}

In this paper we assume that the claims in the surplus process arrive according to a Markovian arrival process (MAP). The underlying environment process, say $\{J(t), t \geq 0\}$, is a continuoustime Markov process with representation $\left\{\boldsymbol{\alpha}, \boldsymbol{D}_{0}, \boldsymbol{D}_{1}\right\}$, where $\boldsymbol{\alpha}$ is the initial probability vector and $\boldsymbol{D}_{0}+\boldsymbol{D}_{1}$ is the intensity matrix. Assume that $J(t)$ is irreducible with finite space $\mathbb{E}=$ $\{1, \ldots, m\}$. The submatrices $\boldsymbol{D}_{0}=\left[D_{0, i j}\right]_{i, j=1}^{m}$ and $\boldsymbol{D}_{1}=\left[D_{1, i j}\right]_{i, j=1}^{m}$ are such that

$$
\begin{gathered}
0 \leq D_{1, i j}<\infty, \quad 0 \leq D_{0, i j}<\infty, \quad i \neq j, \\
D_{0, i i}<0, \quad \sum_{j=1}^{m}\left(D_{0, i j}+D_{1, i j}\right)=0 .
\end{gathered}
$$

Let $\pi=\left(\pi_{1}, \ldots, \pi_{m}\right)$ be the stationary probability row vector of $J(t)$, such that

$$
\pi\left[D_{0}+D_{1}\right]=\mathbf{0}, \quad \pi e=1,
$$

where $\mathbf{0}$ is an $m$-dimensional row vector of 0 s and $\boldsymbol{e}$ is an $m$-dimensional column vector of $1 \mathrm{~s}$.

The submatrices $\boldsymbol{D}_{1}$ and $\boldsymbol{D}_{0}$ respectively denote the intensities of state changes with and without an accompanying claim. Furthermore, assume that the claim size is dependent on

Received 11 May 2010; revision received 30 August 2010.

* Postal address: Department of Statistics and Actuarial Science, Chongqing University, Chongqing, P. R. China.

** Email address: cquzzm@163.com

*** Postal address: Department of Statistics and Actuarial Science, The University of Hong Kong, Pokfulam Road, Hong Kong. 
the Markovian state of $J(t)$ immediately before and after the state transition. Whenever the transition from state $i$ to state $j$ occurs and a claim arrives, the claim size has distribution $F_{i j}=1-\bar{F}_{i j}$, density $f_{i j}$, mean $\mu_{i j}$, and Laplace transform $\hat{f}_{i j}(s)=\int_{0}^{\infty} \mathrm{e}^{-s x} f_{i j}(x) \mathrm{d} x$. Given the initial surplus $u \geq 0$, the risk model is defined as $U_{\infty}(t)=u+Y(t)$ with

$$
Y(t)=c t-\sum_{i=1}^{N(t)} X_{i},
$$

where $c>0$ is the premium rate, $\{N(t), t \geq 0\}$ is the claim number process, and $\left\{X_{n}\right\}_{n \geq 1}$ is a sequence of claim size random variables taking positive values. The bivariate Markov process $\{(J(t), N(t)), t \geq 0\}$ is called a MAP, and, accordingly, the risk model $U_{\infty}(t)$ is called the MAP risk model. In Section XI of Asmussen (2003), the bivariate Markov process $\{(J(t), Y(t)), t \geq 0\}$ is called the Markov additive process. Throughout this paper, we use MAP as an abbreviation of Markovian arrival process, not Markov additive process.

For notational convenience, let

$$
\mathrm{E}_{i}(\cdot)=\mathrm{E}(\cdot \mid J(0)=i), \quad \mathrm{P}_{i}(\cdot)=\mathrm{P}(\cdot \mid J(0)=i) .
$$

By Proposition XI.2.2 of Asmussen (2003), the matrix-valued moment generating function of $Y(t)$ is given by

$$
\mathrm{E}_{i}\left[\mathrm{e}^{s Y(t)} ; J(t)=j\right]=\left[\mathrm{e}^{K(s) t}\right]_{i j}
$$

with the matrix cumulant generating function $\boldsymbol{K}(s)$ given by

$$
\boldsymbol{K}(s)=c s \boldsymbol{I}+\boldsymbol{D}_{0}+\boldsymbol{D}_{1} \circ \hat{\boldsymbol{f}}(s),
$$

where $\boldsymbol{I}$ is the identity matrix, $\hat{\boldsymbol{f}}(s)=\left[\hat{f}_{i j}(s)\right]_{i, j=1}^{m}$. Here, for two matrices $\boldsymbol{A}=\left[A_{i j}\right]$ and $\boldsymbol{B}=\left[B_{i j}\right]$ with the same dimension, $\boldsymbol{A} \circ \boldsymbol{B}=\left[A_{i j} B_{i j}\right]$ denotes the entrywise multiplication. Note that $\boldsymbol{K}(s)$ is well defined at least for $\operatorname{Re}(s) \geq 0$.

The MAP risk model, as an extension of the classical risk model and the Markov-modulated risk model, has received a lot of attention in the last few years. Badescu et al. (2005a), (2005b) studied the ruin probability and the joint distribution of the surplus before and after ruin. Ahn and Badescu (2007) studied the discounted penalty function. Note that in these papers the assumption on the phase-type claim size distribution is important, so that the risk model can be connected to the fluid flow model. For the same MAP risk model, Cheung and Landriault (2010) studied a generalized discounted penalty function incorporating the maximum surplus before ruin.

Recently, more and more researchers have paid attention to the risk model with debit interest. It is assumed that the company does not cease to operate when the traditional ruin occurs, i.e. the surplus drops below level 0 for the first time. The insurer could borrow money at a constant interest rate and then repay the debts continuously from its premium income. Gerber (1971) first considered the absolute ruin probability in the compound Poisson risk model when the debit and credit interest rates are the same; Dassios and Embrechts (1989) studied the absolute ruin probability using a martingale approach and the theory of piecewise deterministic Markov processes; Embrechts and Schmidli (1994) considered the absolute ruin probability in a piecewise-deterministic Markov risk process; Gerber and Yang (2007) considered the absolute ruin probability in a jump-diffusion model with different credit and debit rates. Cai (2007) studied the discounted penalty function at absolute ruin in the classical risk model; Yin and Wang (2010) studied the absolute ruin in a perturbed compound Poisson risk process 
with investment and debit interest. More recently, Konstantinides et al. (2010) studied the asymptotic expressions for the absolute ruin probabilities in a renewal risk model with constant force of interest.

Now consider the same situation as in $U_{\infty}(t)$, but we assume that, whenever the surplus falls below the zero level or the company is in deficit, the insurer could borrow an amount of money equal to the deficit at a debit interest force $r>0$. Under such a modification, we denote the surplus process by $U_{r}(t)$. The mathematical description of $U_{r}(t)$ is

$$
\mathrm{d} U_{r}(t)= \begin{cases}\mathrm{d} Y(t), & U_{r}(t)>0, \\ r U_{r}(t) \mathrm{d} t+\mathrm{d} Y(t), & U_{r}(t)<0 .\end{cases}
$$

Note that, when the surplus is equal to or below the critical level $-c / r$, it will not be able to return to a positive level. Let $T_{r}=\inf \left\{t \geq 0: U_{r}(t) \leq-c / r\right\}$ be the absolute ruin time, where $T_{r}=\infty$ if absolute ruin never occurs in any finite time.

Given the initial environment $J(0)=i$ and the initial surplus $U_{r}(0)=u$, the discounted penalty function is defined as

$$
\Phi_{i j}(u)=\mathrm{E}_{i}\left[\mathrm{e}^{-\delta T_{r}} w\left(U_{r}\left(T_{r}-\right),\left|U_{r}\left(T_{r}\right)\right|\right) \mathbf{1}_{\left\{T_{r}<\infty, J\left(T_{r}\right)=j\right\}} \mid U_{r}(0)=u\right],
$$

where $\delta \geq 0$ is the interest force, $\mathbf{1}_{A}$ is the indicator function of event $A$, and $w:(-c / r, \infty) \times$ $[c / r, \infty) \rightarrow(0, \infty)$ is a measurable penalty function of the surplus immediately before ruin, $U_{r}\left(T_{r}-\right)$, and the deficit at ruin, $\left|U_{r}\left(T_{r}\right)\right|$. Throughout this paper, we assume that $w$ is a bounded function. Furthermore, we assume that the following net profit condition holds:

$$
\sum_{i=1}^{m} \sum_{j=1}^{m} \pi_{i} D_{1, i j} \mu_{i j}<c .
$$

The discounted penalty function was first introduced in Gerber and Shiu (1998) in a classical insurance risk model; it is often called the Gerber-Shiu function.

In the rest of this paper, matrix notation will be frequently used. Write $\boldsymbol{\Phi}(u)=\left[\Phi_{i j}(u)\right]_{i, j=1}^{m}$, $\boldsymbol{f}(x)=\left[f_{i j}(x)\right]_{i, j=1}^{m}$, and $\boldsymbol{F}(x)=\left[F_{i j}(x)\right]_{i, j=1}^{m}$, and $\overline{\boldsymbol{F}}(x)=\left[\bar{F}_{i j}(x)\right]_{i, j=1}^{m}$. For a matrix $\boldsymbol{A}$, we denote its $(i, j)$ th entry by $[\boldsymbol{A}]_{i, j}$, its transpose by $\boldsymbol{A}^{\top}$. For two functions $f_{1}$ and $f_{2}$ supported on $[0, \infty)$, the convolution is defined by

$$
f_{1} * f_{2}(x)=\int_{0}^{x} f_{1}(y) f_{2}(x-y) \mathrm{d} y, \quad x \geq 0 .
$$

For two matrix-valued functions $\boldsymbol{A}_{1}(x)$ and $\boldsymbol{A}_{2}(x)$ with the same dimension, define

$$
\boldsymbol{A}_{1} * \boldsymbol{A}_{2}(x)=\int_{0}^{x} \boldsymbol{A}_{1}(y) \boldsymbol{A}_{2}(x-y) \mathrm{d} y, \quad x \geq 0 .
$$

Furthermore, for $n>1$, let $f^{* n}(x)=f^{*(n-1)} * f(x)$ and $\boldsymbol{A}^{* n}(x)=\boldsymbol{A}^{*(n-1)} * \boldsymbol{A}(x)$. The Laplace transform of a function is denoted with a 'hat' symbol. Note that, for a matrix-valued function $\boldsymbol{A}(x)=\left[A_{i j}(x)\right]_{i, j=1}^{m}, \hat{\boldsymbol{A}}(s)$ means $\left[\hat{A}_{i j}(s)\right]_{i, j=1}^{m}$.

\section{Integro-differential equations and their solutions}

In this section we first derive a system of integro-differential equations with boundary conditions for the discounted penalty functions, and then discuss the solutions. For convenience, let $\Phi_{+, i j}(u)=\Phi_{i j}(u)$ for $u \geq 0$ and $\Phi_{-, i j}(u)=\Phi_{i j}(u)$ for $u<0$. Set $\Phi_{+}(u)=\left[\Phi_{+, i j}(u)\right]_{i, j=1}^{m}$ and $\boldsymbol{\Phi}_{-}(u)=\left[\Phi_{-, i j}(u)\right]_{i, j=1}^{m}$. 
Theorem 1. The discounted penalty functions $\boldsymbol{\Phi}_{+}(u)$ and $\boldsymbol{\Phi}_{-}(u)$ satisfy the following integrodifferential equations: for $u \geq 0$,

$$
\begin{aligned}
\left(\delta \boldsymbol{I}-\boldsymbol{D}_{0}\right) \boldsymbol{\Phi}_{+}(u)= & c \boldsymbol{\Phi}_{+}^{\prime}(u)+\int_{0}^{u}\left[\boldsymbol{D}_{1} \circ \boldsymbol{f}(x)\right] \boldsymbol{\Phi}_{+}(u-x) \mathrm{d} x \\
& +\int_{u}^{u+c / r}\left[\boldsymbol{D}_{1} \circ \boldsymbol{f}(x)\right] \boldsymbol{\Phi}_{-}(u-x) \mathrm{d} x+\boldsymbol{D}_{1} \circ \boldsymbol{\omega}(u)
\end{aligned}
$$

and, for $-c / r<u<0$,

$$
\left(\delta \boldsymbol{I}-\boldsymbol{D}_{0}\right) \boldsymbol{\Phi}_{-}(u)=(u r+c) \boldsymbol{\Phi}_{-}^{\prime}(u)+\int_{0}^{u+c / r}\left[\boldsymbol{D}_{1} \circ \boldsymbol{f}(x)\right] \boldsymbol{\Phi}_{-}(u-x) \mathrm{d} x+\boldsymbol{D}_{1} \circ \boldsymbol{\omega}(u),
$$

where $\boldsymbol{\omega}(u)=\left[\omega_{i j}(u)\right]_{i, j=1}^{m}$ with $\omega_{i j}(u)=\int_{u+c / r}^{\infty} w(u, x-u) f_{i j}(x) \mathrm{d} x$.

Proof. For $u \geq 0$, by conditioning on the time of the first state change of the Markov process $(J(t), N(t))$, we have

$$
\begin{aligned}
\Phi_{+, i j}(u)= & \int_{0}^{\infty} \mathrm{e}^{\left(D_{0, i i}-\delta\right) t} \sum_{k=1, k \neq i}^{m} D_{0, i k} \Phi_{+, k j}(u+c t) \mathrm{d} t \\
& +\int_{0}^{\infty} \mathrm{e}^{\left(D_{0, i i}-\delta\right) t} \sum_{k=1}^{m} D_{1, i k}\left[\int_{0}^{u+c t} \Phi_{+, k j}(u+c t-x) f_{i k}(x) \mathrm{d} x\right. \\
& \left.+\int_{u+c t}^{u+c t+c / r} \Phi_{-, k j}(u+c t-x) f_{i k}(x) \mathrm{d} x\right] \mathrm{d} t \\
& +\int_{0}^{\infty} \mathrm{e}^{\left(D_{0, i i}-\delta\right) t} D_{1, i j} \int_{u+c t+c / r}^{\infty} w(u+c t, x-u-c t) f_{i j}(x) \mathrm{d} x \mathrm{~d} t .
\end{aligned}
$$

Making the change of variable $s=u+c t$, we obtain

$$
\begin{aligned}
\Phi_{+, i j}(u)= & \int_{u}^{\infty} \frac{1}{c} \mathrm{e}^{\left(D_{0, i i}-\delta\right)(s-u) / c} \sum_{k=1, k \neq i}^{m} D_{0, i k} \Phi_{+, k j}(s) \mathrm{d} s \\
& +\int_{u}^{\infty} \frac{1}{c} \mathrm{e}^{\left(D_{0, i i}-\delta\right)(s-u) / c} \sum_{k=1}^{m} D_{1, i k}\left[\int_{0}^{s} \Phi_{+, k j}(s-x) f_{i k}(x) \mathrm{d} x\right. \\
& \left.+\int_{s}^{s+c / r} \Phi_{-, k j}(s-x) f_{i k}(x) \mathrm{d} x\right] \mathrm{d} s \\
& +\int_{u}^{\infty} \frac{1}{c} \mathrm{e}^{\left(D_{0, i i}-\delta\right)(s-u) / c} D_{1, i j} \omega_{i j}(s) \mathrm{d} s .
\end{aligned}
$$

Differentiating both sides of (4) gives

$$
\begin{aligned}
c \Phi_{+, i j}^{\prime}(u)= & \delta \Phi_{+, i j}(u)-\sum_{k=1}^{m} D_{0, i k} \Phi_{+, k j}(u) \\
& -\sum_{k=1}^{m} D_{1, i k}\left[\int_{0}^{u} \Phi_{+, k j}(u-x) f_{i k}(x) \mathrm{d} x+\int_{u}^{u+c / r} \Phi_{-, k j}(u-x) f_{i k}(x) \mathrm{d} x\right] \\
& -D_{1, i j} \omega_{i j}(u) .
\end{aligned}
$$

Equation (2) is the matrix form of the above equation. 
For $-c / r<u<0$, let $t_{0}=(1 / r) \ln c /(u r+c)$, which is the solution of the following equation:

$$
h(u, t):=u \mathrm{e}^{r t}+c \frac{\mathrm{e}^{r t}-1}{r}=0 .
$$

Note that before time $t_{0}$, the surplus process $U_{r}(t)$ stays below level 0 . In particular, for $t<t_{0}$, $U_{r}(t)=h(u, t)$ prior to the first claim arrival. By conditioning on the time of the first state change of the Markov process $(J(t), N(t))$, we have

$$
\begin{aligned}
\Phi_{-, i j}(u)= & \int_{0}^{t_{0}} \mathrm{e}^{\left(D_{0, i i}-\delta\right) t} \sum_{k=1, k \neq i}^{m} D_{0, i k} \Phi_{-, k j}(h(u, t)) \mathrm{d} t \\
& +\int_{0}^{t_{0}} \mathrm{e}^{\left(D_{0, i i}-\delta\right) t} \sum_{k=1}^{m} D_{1, i k} \int_{0}^{h(u, t)+c / r} \Phi_{-, k j}(h(u, t)-x) f_{i k}(x) \mathrm{d} x \mathrm{~d} t \\
& +\int_{0}^{t_{0}} \mathrm{e}^{\left(D_{0, i i}-\delta\right) t} D_{1, i j} \omega_{i j}(h(u, t)) \mathrm{d} t \\
& +\int_{t_{0}}^{\infty} \mathrm{e}^{\left(D_{0, i i}-\delta\right) t} \sum_{k=1, k \neq i}^{m} D_{0, i k} \Phi_{+, k j}\left(c\left(t-t_{0}\right)\right) \mathrm{d} t \\
& +\int_{t_{0}}^{\infty} \mathrm{e}^{\left(D_{0, i i}-\delta\right) t} \sum_{k=1}^{m} D_{1, i k}\left[\int_{0}^{c\left(t-t_{0}\right)} \Phi_{+, k j}\left(c\left(t-t_{0}\right)-x\right) f_{i k}(x) \mathrm{d} x\right. \\
& +\int_{t_{0}}^{\infty} \mathrm{e}^{\left(D_{0, i i}-\delta\right) t} D_{1, i j} \omega_{i j}\left(c\left(t-t_{0}\right)\right) \mathrm{d} t .
\end{aligned}
$$

By changing some variables in (5), we can obtain

$$
\begin{aligned}
\Phi_{-, i j}(u)=\int_{u}^{0} & \frac{1}{s r+c}\left(\frac{s r+c}{u r+c}\right)^{\left(D_{0, i i}-\delta\right) / r} \\
& \times\left[\sum_{k=1, k \neq i}^{m} D_{0, i k} \Phi_{-, k j}(s)\right. \\
& \left.\quad+\sum_{k=1}^{m} D_{1, i k} \int_{0}^{s+c / r} \Phi_{-, k j}(s-x) f_{i k}(x) \mathrm{d} x+D_{1, i j} \omega_{i j}(s)\right] \mathrm{d} s \\
+ & \left(\frac{c}{u r+c}\right)^{\left(D_{0, i i}-\delta\right) / r} \\
& \times \int_{0}^{\infty} \mathrm{e}^{\left(D_{0, i i}-\delta\right) s / c}\left[\sum_{k=1, k \neq i}^{m} D_{0, i k} \Phi_{+, k j}(s)+D_{1, i j} \omega_{i j}(s)\right. \\
& +\sum_{k=1}^{m} D_{1, i k}\left(\int_{0}^{s} \Phi_{+, k j}(s-x) f_{i k}(x) \mathrm{d} x\right. \\
& \\
&
\end{aligned}
$$


Differentiating both sides of (6) with respect to $u$ gives

$$
\begin{aligned}
(u r+c) \Phi_{-, i j}^{\prime}(u)= & \delta \Phi_{-, i j}(u)-\sum_{k=1}^{m} D_{0, i k} \Phi_{-, k j}(u) \\
& -\sum_{k=1}^{m} D_{1, i k} \int_{0}^{u+c / r} \Phi_{-, k j}(u-x) f_{i k}(x) \mathrm{d} x-D_{1, i j} \omega_{i j}(u) .
\end{aligned}
$$

Rewriting the above equation in matrix form gives (3).

We can obtain some boundary conditions from the above derivation procedure. Firstly, from (4) and (6), we have

$$
\boldsymbol{\Phi}_{+}(0)=\boldsymbol{\Phi}_{-}(0-)
$$

Rewrite (6) as

$$
\Phi_{-, i j}(u)=\frac{\int_{u}^{0}(s r+c)^{\left(D_{0, i i}-\delta\right) / r-1} W_{1, i j}(s) \mathrm{d} s}{(u r+c)^{\left(D_{0, i i}-\delta\right) / r}}+\left(\frac{c}{u r+c}\right)^{\left(D_{0, i i}-\delta\right) / r} W_{2, i j},
$$

where

$$
\begin{aligned}
W_{1, i j}(s)= & \sum_{\substack{k=1, k \neq i \\
m}}^{m} D_{0, i k} \Phi_{-, k j}(s)+\sum_{k=1}^{m} D_{1, i k} \int_{0}^{s+c / r} \Phi_{-, k j}(s-x) f_{i k}(x) \mathrm{d} x \\
& +D_{1, i j} \omega_{i j}(s), \\
W_{2, i j}= & \int_{0}^{\infty} \mathrm{e}^{\left(D_{0, i i}-\delta\right) s / c}\left[\sum_{k=1, k \neq i}^{m} D_{0, i k} \Phi_{+, k j}(s)+D_{1, i j} \omega_{i j}(s)\right. \\
& +\sum_{k=1}^{m} D_{1, i k}\left(\int_{0}^{s} \Phi_{+, k j}(s-x) f_{i k}(x) \mathrm{d} x\right. \\
& \left.\left.+\int_{s}^{s+c / r} \Phi_{-, k j}(s-x) f_{i k}(x) \mathrm{d} x\right)\right] \mathrm{d} s .
\end{aligned}
$$

Because $w$ is a bounded function, it is not hard to see that $W_{2, i j}<\infty$. Furthermore, if

$$
\lim _{u \downarrow-c / r} \int_{u}^{0}(s r+c)^{\left(D_{0, i i}-\delta\right) / r-1} \omega_{i j}(s) \mathrm{d} s=\infty, \quad i, j \in \mathcal{E},
$$

then

$$
\lim _{u \downarrow-c / r} \int_{u}^{0}(s r+c)^{\left(D_{0, i i}-\delta\right) / r-1} W_{1, i j}(s) \mathrm{d} s=\infty .
$$

In this case, by l'Hôpital's rule, we have

$$
\begin{aligned}
\Phi_{-, i j}\left(-\frac{c}{r}\right)= & \lim _{u \downarrow-c / r} \frac{\int_{u}^{0}(s r+c)^{\left(D_{0, i i}-\delta\right) / r-1} W_{1, i j}(s) \mathrm{d} s}{(u r+c)^{\left(D_{0, i i}-\delta\right) / r}} \\
& +\lim _{u \downarrow-c / r}\left(\frac{c}{u r+c}\right)^{\left(D_{0, i i}-\delta\right) / r} W_{2, i j}(s) \\
= & \frac{-W_{1, i j}(-c / r)}{D_{0, i i}-\delta},
\end{aligned}
$$


that is, for $i, j \in \mathcal{E}$,

$$
\sum_{k=1}^{m} D_{0, i k} \Phi_{-, k j}\left(-\frac{c}{r}\right)-\delta \Phi_{-, i j}\left(-\frac{c}{r}\right)+D_{1, i j} \omega_{i j}\left(-\frac{c}{r}\right)=0 .
$$

Rewriting the above equation in matrix form gives

$$
\left[\delta \boldsymbol{I}-\boldsymbol{D}_{0}\right] \boldsymbol{\Phi}_{-}\left(-\frac{c}{r}\right)=\boldsymbol{D}_{1} \circ \omega\left(-\frac{c}{r}\right) .
$$

Since $\boldsymbol{D}_{0}$ is a subgenerator matrix, $\delta \boldsymbol{I}-\boldsymbol{D}_{0}$ is nonsingular. Then

$$
\boldsymbol{\Phi}_{-}\left(-\frac{c}{r}\right)=\left[\delta \boldsymbol{I}-\boldsymbol{D}_{0}\right]^{-1}\left[\boldsymbol{D}_{1} \circ \omega\left(-\frac{c}{r}\right)\right] .
$$

We remark that most of the penalty functions used in ruin theory satisfy (8).

Now we discuss the solutions to (2) and (3). Firstly, we consider (3). Note that

$$
\begin{aligned}
\int_{-c / r}^{u} & \int_{0}^{t+c / r}\left[\boldsymbol{D}_{1} \circ \boldsymbol{f}(x)\right] \boldsymbol{\Phi}_{-}(t-x) \mathrm{d} x \mathrm{~d} t \\
& =\int_{-c / r}^{u} \int_{-c / r}^{t}\left[\boldsymbol{D}_{1} \circ \boldsymbol{f}(t-x)\right] \boldsymbol{\Phi}_{-}(x) \mathrm{d} x \mathrm{~d} t \\
& =\int_{-c / r}^{u} \int_{x}^{u}\left[\boldsymbol{D}_{1} \circ \boldsymbol{f}(t-x)\right] \mathrm{d} t \boldsymbol{\Phi}_{-}(x) \mathrm{d} x \\
& =\int_{-c / r}^{u}\left[\boldsymbol{D}_{1} \circ \boldsymbol{F}(u-x)\right] \boldsymbol{\Phi}_{-}(x) \mathrm{d} x .
\end{aligned}
$$

Then replacing $u$ in (3) by $t$ and integrating both sides from $-c / r$ to $u$ gives

$$
\boldsymbol{\Phi}_{-}(u)=\int_{-c / r}^{u} \boldsymbol{K}_{-}(u, x) \boldsymbol{\Phi}_{-}(x) \mathrm{d} x+\boldsymbol{H}_{-}(u), \quad-\frac{c}{r}<u<0,
$$

where

$$
\begin{aligned}
\boldsymbol{K}_{-}(u, x) & =\frac{(\delta+r) \boldsymbol{I}-\boldsymbol{D}_{0}-\left[\boldsymbol{D}_{1} \circ \boldsymbol{F}(u-x)\right]}{u r+c}, \\
\boldsymbol{H}_{-}(u) & =-\frac{1}{u r+c} \int_{-c / r}^{u}\left[\boldsymbol{D}_{1} \circ \boldsymbol{\omega}(t)\right] \mathrm{d} t .
\end{aligned}
$$

Under some regular conditions, for example, the penalty function $w$ is bounded, we can obtain

$$
\begin{aligned}
\lim _{u \downarrow-c / r} \boldsymbol{\Phi}_{-}(u) & =\lim _{u \downarrow-c / r} \int_{-c / r}^{u} \boldsymbol{K}_{-}(u, x) \boldsymbol{\Phi}_{-}(x) \mathrm{d} x+\lim _{u \downarrow-c / r} \boldsymbol{H}_{-}(u) \\
& =\frac{1}{r}\left[(\delta+r) \boldsymbol{I}-\boldsymbol{D}_{0}\right] \boldsymbol{\Phi}_{-}\left(-\frac{c}{r}\right)-\frac{1}{r} \boldsymbol{D}_{1} \circ \omega\left(-\frac{c}{r}\right)
\end{aligned}
$$

thanks to l'Hôpital's rule, which will recover boundary condition (9) again after some rearrangement. 
Equation (10) is a matrix Volterra integral equation of the second kind. Obviously, $\boldsymbol{H}_{-}$ is absolutely integrable and the kernel $\boldsymbol{K}_{-}$is continuous. Then $\boldsymbol{\Phi}_{-}(u)$ can be approximated recursively by Picard's sequence $\left\{\boldsymbol{\Phi}_{n,-}(u), n \geq 0\right\}$, where $\boldsymbol{\Phi}_{0,-}(u)=\boldsymbol{H}_{-}(u)$, and, for $n \geq 1$,

$$
\boldsymbol{\Phi}_{n,-}(u)=\int_{-c / r}^{u} \boldsymbol{K}_{-}(u, x) \boldsymbol{\Phi}_{n-1,-}(x) \mathrm{d} x+\boldsymbol{H}_{-}(u) .
$$

We cannot get the desired explicit expression for $\boldsymbol{\Phi}_{-}(u)$ by (11). However, we can adopt some numerical approach to approximate $\boldsymbol{\Phi}_{-}(u)$ at some lattice points. In particular, with boundary condition (9) in hand, this problem can be reduced to solving some linear system of algebraic equations. We refer the readers to Linz (1985) for the solution procedure, where many methods of solving the Volterra integral equations are presented.

Next, we consider (2). Let $\boldsymbol{V}(u)$ be the solution of the homogeneous integro-differential equation

$$
\left(\delta \boldsymbol{I}-\boldsymbol{D}_{0}\right) \boldsymbol{V}(u)=c \boldsymbol{V}^{\prime}(u)+\int_{0}^{u}\left[\boldsymbol{D}_{1} \circ \boldsymbol{f}(x)\right] \boldsymbol{V}(u-x) \mathrm{d} x
$$

with initial condition $\boldsymbol{V}(0)=\boldsymbol{I}$. Then by the general theory of differential equations we have

$$
\begin{aligned}
\boldsymbol{\Phi}_{+}(u) & =\boldsymbol{V}(u) \boldsymbol{\Phi}_{+}(0)-\frac{1}{c} \int_{0}^{u} \boldsymbol{V}(x) \boldsymbol{B}_{r}(u-x) \mathrm{d} x \\
& =\boldsymbol{V}(u) \boldsymbol{\Phi}_{-}(0)-\frac{1}{c} \int_{0}^{u} \boldsymbol{V}(x) \boldsymbol{B}_{r}(u-x) \mathrm{d} x,
\end{aligned}
$$

due to the continuity condition (7), where

$$
\boldsymbol{B}_{r}(u)=\int_{u}^{u+c / r}\left[\boldsymbol{D}_{1} \circ \boldsymbol{f}(x)\right] \boldsymbol{\Phi}_{-}(u-x) \mathrm{d} x+\boldsymbol{D}_{1} \circ \boldsymbol{\omega}(u) .
$$

From (12), we know that $\boldsymbol{\Phi}_{+}(u)$ is heavily dependent on the functions $\boldsymbol{V}(u)$ and $\boldsymbol{\Phi}_{-}(u)$. As remarked in Cheung and Landriault (2010), if the elements in the Laplace transform $\hat{\boldsymbol{f}}(s)$ are rational then the elements in the Laplace transform $\hat{\boldsymbol{V}}(s)$ are also rational. In this case, $\boldsymbol{V}(u)$ can be readily obtained by inverting the Laplace transforms.

We can also rewrite (12) as

$$
\begin{aligned}
\boldsymbol{\Phi}_{+}(u)= & \boldsymbol{V}(u) \boldsymbol{\Phi}_{-}(0)-\frac{1}{c} \int_{-c / r}^{0} \int_{0}^{u} \boldsymbol{V}(u-x)\left[\boldsymbol{D}_{1} \circ \boldsymbol{f}(x-t)\right] \mathrm{d} x \boldsymbol{\Phi}_{-}(t) \mathrm{d} t \\
& -\frac{1}{c} \int_{0}^{u} \boldsymbol{V}(u-x)\left[\boldsymbol{D}_{1} \circ \boldsymbol{\omega}(x)\right] \mathrm{d} x .
\end{aligned}
$$

Thus, we can first get the approximative values of $\boldsymbol{\Phi}_{-}(t)$ at some lattice points $-c / r=t_{0}<$ $t_{1}<\cdots<t_{n-1}<t_{n}=0$, and then apply (13) to approximate $\boldsymbol{\Phi}_{+}(u)$ by some numerical integration methods.

The above arguments show that it is feasible to calculate the discounted penalty functions by some numerical methods. However, due to the difficulty of finding the explicit expressions, asymptotic results become significant and interesting.

\section{A matrix renewal equation for $\Phi_{+}(u)$}

In this section we derive a matrix renewal equation for $\boldsymbol{\Phi}_{+}(u)$ that is useful in studying the asymptotic behavior of the discounted penalty function. Firstly, we present some preliminaries that are due to Breuer (2008). 
Consider a bivariate process $(\tilde{J}, \tilde{Y})$ which represents the time reversed process $(J, Y)$ from a fixed time in the future when $J$ starts from the stationary distribution $\pi$. That is,

$$
\tilde{J}(s)=J((t-s)-), \quad \tilde{Y}(s)=Y(t)-Y((t-s)-), \quad 0 \leq s \leq t,
$$

under $\mathrm{P}_{\pi}:=\sum_{i \in \mathcal{E}} \pi_{i} \mathrm{P}_{i}$. The bivariate process $(\tilde{J}, \tilde{Y})$ is still a Markov additive process. In the sequel, we will use a tilde to indicate the characteristics associated with $(\tilde{J}, \tilde{Y})$. The intensity matrix $\tilde{\boldsymbol{D}}_{0}+\tilde{\boldsymbol{D}}_{1}$ must satisfy

$$
\tilde{\boldsymbol{D}}_{0}=\boldsymbol{\Delta}_{\pi}^{-1} \boldsymbol{D}_{0}^{\top} \boldsymbol{\Delta}_{\pi}, \quad \tilde{\boldsymbol{D}}_{1}=\boldsymbol{\Delta}_{\pi}^{-1} \boldsymbol{D}_{1}^{\top} \boldsymbol{\Delta}_{\pi},
$$

with $\boldsymbol{\Delta}_{\pi}=\operatorname{diag}\left(\pi_{1}, \ldots, \pi_{m}\right)$. The matrix cumulant generating function associated with $\tilde{Y}$ is given by

$$
\tilde{\boldsymbol{K}}(s)=\boldsymbol{\Delta}_{\boldsymbol{\pi}}^{-1} \boldsymbol{K}(s)^{\top} \boldsymbol{\Delta}_{\boldsymbol{\pi}} .
$$

In the rest of the paper, whenever we talk about the process $(\tilde{J}, \tilde{Y})$, we will refer to it under the probabilities $\left\{\tilde{\mathrm{P}}_{i}: i \in \mathcal{E}\right\}$.

For $x \geq 0$, let $\tau_{x}^{+}=\inf \{t \geq 0: Y(t)=x\}$ be the first time when $Y$ reaches the level $x$. Due to the net profit condition (1), $\left\{\tau_{x}^{+}, x \geq 0\right\}$ is a nonterminating continuous-time Markov process. From Section 3 of Breuer (2008), we know that there exists a matrix $\boldsymbol{Q}_{\delta}$ such that, for $\delta \geq 0$,

$$
\tilde{\mathrm{E}}_{i}\left[\mathrm{e}^{-\delta \tau_{x}^{+}} ; J\left(\tau_{x}^{+}\right)=j\right]=\left[\mathrm{e}^{\boldsymbol{Q}_{\delta x}}\right]_{i j} .
$$

Under the probabilities $\left\{\tilde{\mathrm{P}}_{i}: i \in \mathcal{E}\right\}, \boldsymbol{Q}_{0}$ is the generator matrix of $\left\{\tau_{x}^{+}\right\}$, whereas, for $\delta>0$, $\boldsymbol{Q}_{\delta}$ is a subgenerator matrix. Thus, all eigenvalues of $\boldsymbol{Q}_{\delta}$ are in the left-half complex plane. By Theorem 1 of Breuer (2008) we know that $\boldsymbol{Q}_{\delta}$ satisfies the following nonlinear matrix equation:

$$
c \boldsymbol{Q}_{\delta}=\tilde{\boldsymbol{D}}_{0}-\delta \boldsymbol{I}+\int_{0}^{\infty}\left[\tilde{\boldsymbol{D}}_{1} \circ \hat{\boldsymbol{f}}(x)\right] \mathrm{e}^{\boldsymbol{Q}_{\delta} x} \mathrm{~d} x .
$$

Furthermore, Theorem 2 of Breuer (2008) states that $\boldsymbol{Q}_{\delta}$ can be computed as the limit of the sequence $\left\{\boldsymbol{Q}_{\delta, n}, n \geq 0\right\}$, where $\boldsymbol{Q}_{\delta, 0}=(1 / c)\left[\tilde{\boldsymbol{D}}_{0}-\delta \boldsymbol{I}\right]$, and, for $n \geq 1$,

$$
c \boldsymbol{Q}_{\delta, n}=\tilde{\boldsymbol{D}}_{0}-\delta \boldsymbol{I}+\int_{0}^{\infty}\left[\tilde{\boldsymbol{D}}_{1} \circ \hat{\boldsymbol{f}}(x)\right] \mathrm{e}^{\boldsymbol{Q}_{\delta, n-1} x} \mathrm{~d} x .
$$

As will be seen later, it is more convenient for us to consider the matrix $\boldsymbol{P}_{\delta}:=-\left[\boldsymbol{\Delta}_{\pi} \boldsymbol{Q}_{\delta} \boldsymbol{\Delta}_{\pi}^{-1}\right]^{\top}$. Rewriting (15) in terms of $\boldsymbol{P}_{\delta}$ gives

$$
c \boldsymbol{P}_{\delta}=\delta \boldsymbol{I}-\boldsymbol{D}_{0}-\int_{0}^{\infty} \mathrm{e}^{-\boldsymbol{P}_{\delta} x}\left[\boldsymbol{D}_{1} \circ \boldsymbol{f}(x)\right] \mathrm{d} x .
$$

While from (16) we know that $\boldsymbol{P}_{\delta}$ can be approximated by the sequence $\left\{\boldsymbol{P}_{\delta, n}, n \geq 0\right\}$, where $\boldsymbol{P}_{\delta, 0}=(1 / c)\left[\delta \boldsymbol{I}-\boldsymbol{D}_{0}\right]$, and, for $n \geq 1$,

$$
c \boldsymbol{P}_{\delta, n}=\delta \boldsymbol{I}-\boldsymbol{D}_{0}-\int_{0}^{\infty} \mathrm{e}^{-\boldsymbol{P}_{\delta, n-1} x}\left[\boldsymbol{D}_{1} \circ \boldsymbol{f}(x)\right] \mathrm{d} x .
$$

Furthermore, the following lemma shows that $\boldsymbol{P}_{\delta}$ can also be obtained by diagonalization under some conditions. 
Lemma 1. Let $\rho_{\delta, 1}, \ldots, \rho_{\delta, m}$ be the eigenvalues of $\boldsymbol{P}_{\delta}$, and respectively denote by

$$
\boldsymbol{\Delta}_{\rho_{\delta}}=\operatorname{diag}\left(\rho_{\delta, 1}, \ldots, \rho_{\delta, m}\right), \quad \boldsymbol{\Gamma}_{\delta}=\left[\begin{array}{c}
\boldsymbol{\gamma}_{\delta, 1} \\
\vdots \\
\boldsymbol{\gamma}_{\delta, n}
\end{array}\right]
$$

the eigenvalues matrix and the left eigenvectors matrix of $\boldsymbol{P}_{\delta}$. If the eigenvalues are distinct then $\boldsymbol{P}_{\delta}$ has a diagonalized form, $\boldsymbol{P}_{\delta}=\boldsymbol{\Gamma}_{\delta}^{-1} \boldsymbol{\Delta}_{\rho_{\delta}} \boldsymbol{\Gamma}_{\delta}$. Furthermore, the $\rho_{\delta, i}$ s are the roots of the equation

$$
\operatorname{det}[\boldsymbol{K}(s)-\delta \boldsymbol{I}]=0,
$$

and the eigenvectors, $\boldsymbol{\gamma}_{\delta, i}$, can be obtained by solving the equations

$$
\boldsymbol{\gamma}_{\delta, i}\left[\boldsymbol{K}\left(\rho_{\delta, i}\right)-\delta \boldsymbol{I}\right]=\mathbf{0}, \quad i=1, \ldots, m .
$$

Proof. Obviously, if the eigenvalues are distinct then the eigenvectors are nonsingular and $\boldsymbol{P}_{\delta}$ can be diagonalized as $\boldsymbol{P}_{\delta}=\boldsymbol{\Gamma}_{\delta}^{-1} \boldsymbol{\Delta}_{\rho_{\delta}} \boldsymbol{\Gamma}_{\delta}$. Since $\boldsymbol{P}_{\delta}$ satisfies (17), we have

$$
c \boldsymbol{\Gamma}_{\delta}^{-1} \boldsymbol{\Delta}_{\rho_{\delta}} \boldsymbol{\Gamma}_{\delta}=\delta \boldsymbol{I}-\boldsymbol{D}_{0}-\boldsymbol{\Gamma}_{\delta}^{-1} \int_{0}^{\infty} \mathrm{e}^{-\boldsymbol{\Delta}_{\rho_{\delta} x}} \boldsymbol{\Gamma}_{\delta}\left[\boldsymbol{D}_{1} \circ \boldsymbol{f}(x)\right] \mathrm{d} x .
$$

Premultipling the above equation by $\boldsymbol{\Gamma}_{\delta}$ gives

$$
c \boldsymbol{\Delta}_{\rho_{\delta}} \boldsymbol{\Gamma}_{\delta}=\delta \boldsymbol{\Gamma}_{\delta}-\boldsymbol{\Gamma}_{\delta} \boldsymbol{D}_{0}-\int_{0}^{\infty} \mathrm{e}^{-\boldsymbol{\Delta}_{\rho_{\delta}} x} \boldsymbol{\Gamma}_{\delta}\left[\boldsymbol{D}_{1} \circ \boldsymbol{f}(x)\right] \mathrm{d} x,
$$

which is equivalent to (19). Finally, (19) implies that the $\rho_{\delta, i} \mathrm{~s}$ are roots of (18).

Remark 1. Since $\boldsymbol{Q}_{\delta}$ is a (sub)generator matrix and $\boldsymbol{P}_{\delta}$ is similar to $-\boldsymbol{Q}_{\delta}^{\top}$, then the eigenvalues, $\rho_{\delta, i}$, must be in the right-half complex plane. Furthermore, by the Perron-Frobenius theorem (see, e.g. Corollary A4.8 of Asmussen (2000)), we know that the eigenvalue of $\boldsymbol{P}_{\delta}$ with the minimum real part, say $\rho_{\delta}$, is real and strictly less than other eigenvalues. Let $\boldsymbol{\gamma}_{\delta}$ and $\boldsymbol{h}_{\delta}$ be the associated left and right eigenvectors normalized by $\boldsymbol{\gamma}_{\delta} \boldsymbol{h}_{\delta}=1$ and $\boldsymbol{\gamma}_{\delta} \boldsymbol{e}=1$. Then all components of $\boldsymbol{\gamma}_{\delta}$ and $\boldsymbol{h}_{\delta}$ are real and positive. In particular, when $\delta=0$, we have $\rho_{0}=0$ and $\boldsymbol{\gamma}_{0}=\boldsymbol{\pi}$. In the rest of this paper, we will denote $\boldsymbol{h}_{0}$ by $\boldsymbol{h}$.

The following theorem is the main result of this section.

Theorem 2. The discounted penalty function $\boldsymbol{\Phi}_{+}(u)$ satisfies the matrix renewal equation

$$
\boldsymbol{\Phi}_{+}(u)=\int_{0}^{u} \boldsymbol{g}_{\delta}(y) \boldsymbol{\Phi}_{+}(u-y) \mathrm{d} y+Z_{\delta}(u),
$$

where

$$
\begin{aligned}
& \boldsymbol{g}_{\delta}(y)=\frac{1}{c} \int_{0}^{\infty} \mathrm{e}^{-\boldsymbol{P}_{\delta} x}\left[\boldsymbol{D}_{1} \circ \boldsymbol{f}(x+y)\right] \mathrm{d} x, \\
& \boldsymbol{Z}_{\delta}(u)=\frac{1}{c} \int_{0}^{\infty} \mathrm{e}^{-\boldsymbol{P}_{\delta} x} \boldsymbol{B}_{r}(x+u) \mathrm{d} x .
\end{aligned}
$$

Remark 2. Equation (20) is a generalization of the defective renewal equation (14) of Cai (2007), where only the case $m=1$ and $\delta=0$ is considered. We will present two approaches to derive (20). The first approach is analytic, which is based on the assumption that the eigenvalues are distinct. While the second approach is based on some purely probabilistic arguments without any assumption on the eigenvalues. It seems that the second approach is more interesting, but the first approach is practical because in almost all the applications the eigenvalues are distinct. 
Proof of Theorem 2 (analytic approach). In this proof we assume that the eigenvalues, $\rho_{\delta, i}$, are distinct. We start from the integro-differential equation (2). Taking Laplace transforms on both sides of (2) gives

$$
[\boldsymbol{K}(s)-\delta \boldsymbol{I}] \hat{\boldsymbol{\Phi}}_{+}(s)=c \boldsymbol{\Phi}_{+}(0)-\hat{\boldsymbol{B}}_{r}(s)
$$

Note that the matrix $s \boldsymbol{I}-\boldsymbol{P}_{\delta}$ is nonsingular for $s \neq \rho_{\delta, 1}, \ldots, \rho_{\delta, m}$. Since $\boldsymbol{P}_{\delta}$ satisfies (17), for $s \neq \rho_{\delta, 1}, \ldots, \rho_{\delta, m}$, we have

$$
\begin{aligned}
\boldsymbol{K}(s)-\delta \boldsymbol{I}= & c s \boldsymbol{I}-\delta \boldsymbol{I}+\boldsymbol{D}_{0}+\int_{0}^{\infty} \mathrm{e}^{-s \boldsymbol{I} x}\left[\boldsymbol{D}_{1} \circ \boldsymbol{f}(x)\right] \mathrm{d} x \\
& -\left[c \boldsymbol{P}_{\delta}-\delta \boldsymbol{I}+\boldsymbol{D}_{0}+\int_{0}^{\infty} \mathrm{e}^{-\boldsymbol{P}_{\delta} x}\left[\boldsymbol{D}_{1} \circ \boldsymbol{f}(x)\right] \mathrm{d} x\right] \\
= & \left(s \boldsymbol{I}-\boldsymbol{P}_{\delta}\right)\left[c \boldsymbol{I}-\left(\boldsymbol{P}_{\delta}-s \boldsymbol{I}\right)^{-1} \int_{0}^{\infty}\left(\mathrm{e}^{-s \boldsymbol{I} x}-\mathrm{e}^{-\boldsymbol{P}_{\delta} x}\right)\left[\boldsymbol{D}_{1} \circ \boldsymbol{f}(x)\right] \mathrm{d} x\right] \\
= & \left(s \boldsymbol{I}-\boldsymbol{P}_{\delta}\right)\left[c \boldsymbol{I}-\left(\boldsymbol{P}_{\delta}-s \boldsymbol{I}\right)^{-1} \int_{0}^{\infty}\left(\mathrm{e}^{-\left(s \boldsymbol{I}-\boldsymbol{P}_{\delta}\right) x}-\boldsymbol{I}\right) \mathrm{e}^{-\boldsymbol{P}_{\delta} x}\left[\boldsymbol{D}_{1} \circ \boldsymbol{f}(x)\right] \mathrm{d} x\right] \\
= & \left(s \boldsymbol{I}-\boldsymbol{P}_{\delta}\right)\left[c \boldsymbol{I}-\int_{0}^{\infty} \int_{0}^{x} \mathrm{e}^{-\left(s \boldsymbol{I}-\boldsymbol{P}_{\delta}\right) y} \mathrm{~d} y \mathrm{e}^{-\boldsymbol{P}_{\delta} x}\left[\boldsymbol{D}_{1} \circ \boldsymbol{f}(x)\right] \mathrm{d} x\right] \\
= & \left(s \boldsymbol{I}-\boldsymbol{P}_{\delta}\right)\left[c \boldsymbol{I}-\int_{0}^{\infty} \mathrm{e}^{-s y} \int_{y}^{\infty} \mathrm{e}^{-\boldsymbol{P}_{\delta}(x-y)}\left[\boldsymbol{D}_{1} \circ \boldsymbol{f}(x)\right] \mathrm{d} x \mathrm{~d} y\right] .
\end{aligned}
$$

Setting $s=\rho_{\delta, i}$ in (21) and then premultiplying both sides by $\boldsymbol{\gamma}_{\delta, i}$ gives

$$
c \boldsymbol{\gamma}_{\delta, i} \boldsymbol{\Phi}_{+}(0)=\boldsymbol{\gamma}_{\delta, i} \hat{\boldsymbol{B}}_{r}\left(\rho_{\delta, i}\right)=\int_{0}^{\infty} \mathrm{e}^{-\rho_{\delta, i} x} \boldsymbol{\gamma}_{\delta, i} \boldsymbol{B}_{r}(x) \mathrm{d} x, \quad i=1, \ldots, m .
$$

Rewriting the above equations in matrix form gives

$$
c \boldsymbol{\Gamma}_{\delta} \boldsymbol{\Phi}_{+}(0)=\int_{0}^{\infty} \mathrm{e}^{-\boldsymbol{\Delta}_{\rho_{\delta}} x} \boldsymbol{\Gamma}_{\delta} \boldsymbol{B}_{r}(x) \mathrm{d} x .
$$

Hence,

$$
c \boldsymbol{\Phi}_{+}(0)=\boldsymbol{\Gamma}_{\delta}^{-1} \int_{0}^{\infty} \mathrm{e}^{-\boldsymbol{\Delta}_{\rho_{\delta}} x} \boldsymbol{\Gamma}_{\delta} \boldsymbol{B}_{r}(x) \mathrm{d} x=\int_{0}^{\infty} \mathrm{e}^{-\boldsymbol{P}_{\delta} x} \boldsymbol{B}_{r}(x) \mathrm{d} x .
$$

By (23) we have

$$
\begin{aligned}
c \boldsymbol{\Phi}_{+}(0)-\hat{\boldsymbol{B}}_{r}(s) & =\int_{0}^{\infty} \mathrm{e}^{-\boldsymbol{P}_{\delta} x} \boldsymbol{B}_{r}(x) \mathrm{d} x-\int_{0}^{\infty} \mathrm{e}^{-s x} \boldsymbol{B}_{r}(x) \mathrm{d} x \\
& =\left(s \boldsymbol{I}-\boldsymbol{P}_{\delta}\right)\left(s \boldsymbol{I}-\boldsymbol{P}_{\delta}\right)^{-1} \int_{0}^{\infty}\left[\boldsymbol{I}-\mathrm{e}^{-\left(s \boldsymbol{I}-\boldsymbol{P}_{\delta}\right) x}\right] \mathrm{e}^{-\boldsymbol{P}_{\delta} x} \boldsymbol{B}_{r}(x) \mathrm{d} x \\
& =\left(s \boldsymbol{I}-\boldsymbol{P}_{\delta}\right) \int_{0}^{\infty} \int_{0}^{x} \mathrm{e}^{-\left(s \boldsymbol{I}-\boldsymbol{P}_{\delta}\right) u} \mathrm{~d} u \mathrm{e}^{-\boldsymbol{P}_{\delta} x} \boldsymbol{B}_{r}(x) \mathrm{d} x \\
& =\left(s \boldsymbol{I}-\boldsymbol{P}_{\delta}\right) \int_{0}^{\infty} \mathrm{e}^{-s u} \int_{u}^{\infty} \mathrm{e}^{-\boldsymbol{P}_{\delta}(x-u)} \boldsymbol{B}_{r}(x) \mathrm{d} x \mathrm{~d} u,
\end{aligned}
$$


which together with (21) and (22) gives

$$
\begin{aligned}
{[c \boldsymbol{I}} & \left.-\int_{0}^{\infty} \mathrm{e}^{-s y} \int_{y}^{\infty} \mathrm{e}^{-\boldsymbol{P}_{\delta}(x-y)}\left[\boldsymbol{D}_{1} \circ \boldsymbol{f}(x)\right] \mathrm{d} x \mathrm{~d} y\right] \hat{\boldsymbol{\Phi}}_{+}(s) \\
& =\int_{0}^{\infty} \mathrm{e}^{-s u} \int_{u}^{\infty} \mathrm{e}^{-\boldsymbol{P}_{\delta}(x-u)} \boldsymbol{B}_{r}(x) \mathrm{d} x \mathrm{~d} u,
\end{aligned}
$$

where $s \neq \rho_{\delta, 1}, \ldots, \rho_{\delta, m}$. By analytic continuation, (24) holds for all $s$ in the right-half complex plane. Finally, inverting the Laplace transforms in (24) gives (20).

Proof of Theorem 2 (probabilistic approach). Let $\tau_{0}^{-}=\inf \{t \geq 0: Y(t)<0\}$ be the first time when $Y(t)$ drops below the level 0 . Given the initial Markovian state $J(0)=i$, let $f_{i j}(t, x, y)$ be the joint density of $\tau_{0}^{-}, Y\left(\tau_{0}^{-}-\right)$, and $-Y\left(\tau_{0}^{-}\right)$, assuming that the Markovian state at time $\tau_{0}^{-}$is $j$. For $u>0$, consider the following situations when the process $U_{r}(t)$ first drops below the initial surplus $u$ :

(a) if the overshoot, $-Y\left(\tau_{0}^{-}\right)$, is less than $u$ then the surplus stays above the level 0 and the company does not borrow money;

(b) if the overshoot is greater than $u$ but less than $u+c / r$, then the surplus becomes negative and the company will borrow money to repay the claims;

(c) if the overshoot is larger than $u+c / r$, absolute ruin occurs and the surplus immediately before the absolute ruin and the deficit at absolute ruin are respectively $u+Y\left(\tau_{0}^{-}-\right)$and $-Y\left(\tau_{0}^{-}\right)-u$.

Distinguishing the above three situations, for $i, j \in \mathcal{E}$, we have

$$
\begin{aligned}
\Phi_{+, i j}(u)= & \sum_{k=1}^{m} \int_{0}^{\infty} \int_{0}^{\infty} \int_{0}^{u} \mathrm{e}^{-\delta t} f_{i k}(t, x, y) \Phi_{+, k j}(u-y) \mathrm{d} y \mathrm{~d} x \mathrm{~d} t \\
& +\sum_{k=1}^{m} \int_{0}^{\infty} \int_{0}^{\infty} \int_{u}^{u+c / r} \mathrm{e}^{-\delta t} f_{i k}(t, x, y) \Phi_{-, k j}(u-y) \mathrm{d} y \mathrm{~d} x \mathrm{~d} t \\
& +\int_{0}^{\infty} \int_{0}^{\infty} \int_{u+c / r}^{\infty} \mathrm{e}^{-\delta t} f_{i j}(t, x, y) w(x+u, y-u) \mathrm{d} y \mathrm{~d} x \mathrm{~d} t \\
= & \sum_{k=1}^{m} \int_{0}^{\infty} \int_{0}^{u} f_{\delta, i k}(x, y) \Phi_{+, k j}(u-y) \mathrm{d} y \mathrm{~d} x \\
& +\sum_{k=1}^{m} \int_{0}^{\infty} \int_{u}^{u+c / r} f_{\delta, i k}(x, y) \Phi_{-, k j}(u-y) \mathrm{d} y \mathrm{~d} x \\
& +\int_{0}^{\infty} \int_{u+c / r}^{\infty} f_{\delta, i j}(x, y) w(x+u, y-u) \mathrm{d} y \mathrm{~d} x,
\end{aligned}
$$

where $f_{\delta, i j}(x, y)=\int_{0}^{\infty} \mathrm{e}^{-\delta t} f_{i j}(t, x, y) \mathrm{d} t$. Set $\boldsymbol{f}_{\delta}(x, y)=\left[f_{\delta, i j}(x, y)\right]_{i, j=1}^{m}$, and write (25) in the following matrix form:

$$
\begin{aligned}
\boldsymbol{\Phi}_{+}(u)= & \int_{0}^{\infty} \int_{0}^{u} \boldsymbol{f}_{\delta}(x, y) \boldsymbol{\Phi}_{+}(u-y) \mathrm{d} y \mathrm{~d} x+\int_{0}^{\infty} \int_{u}^{u+c / r} \boldsymbol{f}_{\delta}(x, y) \boldsymbol{\Phi}_{-}(u-y) \mathrm{d} y \mathrm{~d} x \\
& +\int_{0}^{\infty} \int_{u+c / r}^{\infty} \boldsymbol{f}_{\delta}(x, y) w(x+u, y-u) \mathrm{d} y \mathrm{~d} x
\end{aligned}
$$


Now we identify $f_{\delta, i j}(x, y)$ for $i, j \in \mathcal{E}$. Conditioning on the Markovian state immediately before time $\tau_{0}^{-}$, we obtain

$$
\begin{aligned}
f_{i j}(x, y) \mathrm{d} x \mathrm{~d} y & \\
= & \int_{t \in(0, \infty)} \mathrm{e}^{-\delta t} \mathrm{P}_{i}\left(\tau_{0}^{-} \in \mathrm{d} t, Y\left(\tau_{0}^{-}-\right) \in \mathrm{d} x,-Y\left(\tau_{0}^{-}\right) \in \mathrm{d} y, J\left(\tau_{0}^{-}\right)=j\right) \\
= & \sum_{k \in \mathcal{E}} \int_{t \in(0, \infty)} \mathrm{e}^{-\delta t} \mathrm{P}_{i}\left(\tau_{0}^{-} \in \mathrm{d} t, Y\left(\tau_{0}^{-}-\right) \in \mathrm{d} x,-Y\left(\tau_{0}^{-}\right) \in \mathrm{d} y,\right. \\
& \left.J\left(\tau_{0}^{-}-\right)=k, J\left(\tau_{0}^{-}\right)=j\right) \\
= & \sum_{k \in \mathcal{E}} \int_{t \in(0, \infty)} \mathrm{e}^{-\delta t} \mathrm{P}_{i}\left(\tau_{0}^{-} \geq t, Y(t-) \in \mathrm{d} x, J(t-)=k\right) D_{1, k j} f_{k j}(x+y) \mathrm{d} t \mathrm{~d} y \\
= & \sum_{k \in \mathcal{E}} \int_{t \in(0, \infty)} \mathrm{e}^{-\delta t} \mathrm{P}_{i}\left(\tau_{0}^{-} \geq t, Y(t) \in \mathrm{d} x, J(t)=k\right) D_{1, k j} f_{k j}(x+y) \mathrm{d} t \mathrm{~d} y \\
:= & \sum_{k \in \mathcal{E}} \mathcal{R}_{i k}^{(\delta)}(\mathrm{d} x) D_{1, k j} f_{k j}(x+y) \mathrm{d} y,
\end{aligned}
$$

where, by time reversal,

$$
\begin{aligned}
\mathcal{R}_{i k}^{(\delta)}(\mathrm{d} x) & =\int_{t \in(0, \infty)} \mathrm{e}^{-\delta t} \mathrm{P}_{i}\left(\tau_{0}^{-} \geq t, Y(t) \in \mathrm{d} x, J(t)=k\right) \mathrm{d} t \\
& =\frac{\pi_{k}}{\pi_{i}} \int_{t \in(0, \infty)} \mathrm{e}^{-\delta t} \tilde{\mathrm{P}}_{k}\left(\tau_{x}^{+} \geq t, Y(t) \in \mathrm{d} x, J(t)=i\right) \mathrm{d} t
\end{aligned}
$$

Note that

$$
\tilde{\mathrm{P}}_{k}\left(\tau_{x}^{+} \geq t, Y(t) \in \mathrm{d} x, J(t)=i\right)=\tilde{\mathrm{P}}_{k}\left(\tau_{x}^{+} \in \mathrm{d} t, J(t)=i\right)
$$

and $\mathrm{d} t=(1 / c) \mathrm{d} x$. We have

$$
\mathcal{R}_{i k}^{(\delta)}(\mathrm{d} x)=\frac{\pi_{k}}{c \pi_{i}} \int_{t \in(0, \infty)} \mathrm{e}^{-\delta t} \tilde{\mathrm{P}}_{k}\left(\tau_{x}^{+} \in \mathrm{d} t, J(t)=i\right) \mathrm{d} x=\frac{\pi_{k}}{c \pi_{i}}\left[\mathrm{e}^{\boldsymbol{Q}_{\delta} x}\right]_{k i} \mathrm{~d} x,
$$

where the second step follows from (14). Substituting (28) into (27) gives

$$
f_{i j}(x, y)=\sum_{k \in \mathcal{E}} \frac{\pi_{k}}{c \pi_{i}}\left[\mathrm{e}^{\boldsymbol{Q}_{\delta} x}\right]_{k i} D_{1, k j} f_{k j}(x+y),
$$

or, in matrix form,

$$
\boldsymbol{f}_{\delta}(x, y)=\frac{1}{c} \mathrm{e}^{-\boldsymbol{P}_{\delta} x}\left[\boldsymbol{D}_{1} \circ \boldsymbol{f}(x+y)\right] .
$$

Finally, substituting (29) into (26), we recover (20).

It is not hard to see that the matrix $\int_{0}^{\infty} \boldsymbol{g}_{\delta}(y) \mathrm{d} y$ is strictly substochastic under either $\delta>0$ or the net profit condition (1). Then the matrix renewal equation (20) has the minimal solution such that, for $u \geq 0$,

$$
\boldsymbol{\Phi}_{+}(u)=Z_{\delta}(u)+\int_{0}^{u} \boldsymbol{\Pi}_{\delta}(y) \boldsymbol{Z}_{\delta}(u-y) \mathrm{d} y,
$$

where $\boldsymbol{\Pi}_{\delta}(y)=\sum_{n=1}^{\infty} \boldsymbol{g}_{\delta}^{* n}(y)$. 


\section{Asymptotic results for heavy-tailed claims}

From Section 2 we know that it is very hard to find explicit expressions for the discounted penalty functions. In this section we will investigate the asymptotic behavior of $\boldsymbol{\Phi}(u)$ when the claim size distributions are heavy tailed. We remark that the asymptotic behavior for the ruin probability in the Markov-modulated risk model has been studied by, e.g. Asmussen et al. (1994), Rolski et al. (1999, pp. 522-525, 549-551), and Asmussen (2000, pp. 269-271).

In the rest of this paper, for two scale-valued functions $a_{1}(x)$ and $a_{2}(x)$, we use $a_{1}(x) \sim$ $a_{2}(x), a_{1}(x) \sim o\left(a_{2}(x)\right)$, and $a_{1}(x) \sim O\left(a_{2}(x)\right)$ to denote $\lim _{x \rightarrow \infty}\left(a_{1}(x) / a_{2}(x)\right)=1$, $\lim _{x \rightarrow \infty}\left(a_{1}(x) / a_{2}(x)\right)=0$, and $0<\liminf _{x \rightarrow \infty}\left(a_{1}(x) / a_{2}(x)\right) \leq \lim \sup _{x \rightarrow \infty}\left(a_{1}(x) / a_{2}(x)\right)$ $<\infty$, respectively. For two matrix-valued functions $\boldsymbol{A}_{1}(x)$ and $\boldsymbol{A}_{2}(x)$, we use $\boldsymbol{A}_{1}(x) \sim$ $\boldsymbol{A}_{2}(x), \boldsymbol{A}_{1}(x) \sim o\left(\boldsymbol{A}_{2}(x)\right)$, and $\boldsymbol{A}_{1}(x) \sim O\left(\boldsymbol{A}_{2}(x)\right)$ to denote $\left[\boldsymbol{A}_{1}(x)\right]_{i j} \sim\left[\boldsymbol{A}_{2}(x)\right]_{i j}$, $\left[\boldsymbol{A}_{1}(x)\right]_{i j} \sim o\left(\left[\boldsymbol{A}_{2}(x)\right]_{i j}\right)$, and $\left[\boldsymbol{A}_{1}(x)\right]_{i j} \sim O\left(\left[\boldsymbol{A}_{2}(x)\right]_{i j}\right)$. Associated with a distribution function $F$ defined on $[0, \infty)$, let $\bar{F}=1-F$ be the tail function and define the convolution $F^{\star 2}(x)=F \star F(x)=\int_{0-}^{x} F(x-y) \mathrm{d} F(y)$.

We introduce some classes of heavy-tailed functions and list some of their properties. Some excellent references on heavy-tailed distributions are Klüppelberg (1988), (1989), Embrechts et al. (1997, pp. 36-57), Asmussen (2000, pp. 251-259), and Asmussen et al. (2003).

Definition 1. A distribution function $F$ on $[0, \infty)$ is said to belong to the class $\mathcal{L}$ of long-tailed distributions if

$$
\lim _{x \rightarrow \infty} \frac{\bar{F}(x-y)}{\bar{F}(x)}=1 \quad \text { for all } y \in \mathbb{R} .
$$

Definition 2. A distribution function $F$ is said to belong to the class $\&$ of subexponential distributions if $F \in \mathcal{L}$ and

$$
\lim _{x \rightarrow \infty} \frac{\overline{F^{\star 2}}(x)}{\bar{F}(x)}=2 .
$$

Definition 3. A measurable function $f:[0, \infty) \rightarrow[0, \infty)$ is said to belong to the class $\mathcal{L}_{d}$ if $f(x)>0$ for all large $x>0$ and

$$
\lim _{x \rightarrow \infty} \frac{f(x-y)}{f(x)}=1 \quad \text { for all } y \in \mathbb{R} .
$$

Definition 4. A measurable function $f$ is said to belong to the class $\varsigma_{d}$ if $f \in \mathcal{L}_{d}$ and the following limit exists:

$$
\lim _{x \rightarrow \infty} \frac{f^{* 2}(x)}{f(x)}=2 d<\infty .
$$

When $f \in \delta_{d}$ is a density function, we call it a subexponential density. It is well known that the classes $\mathcal{L}$ and $\delta$ are closed with respect to tail equivalence, while the classes $\mathcal{L}_{d}$ and $\delta_{d}$ are closed with respect to asymptotic equivalence.

Lemma 2. (Tang and Wei (2010).) If $f \in \mathcal{L}_{d}$ then, for all $\varepsilon>0$, there are some constants $c_{0}, x_{0}>0$ such that, for all $x \geq y \geq x_{0}$,

$$
c_{0}^{-1} \mathrm{e}^{-\varepsilon(x-y)} \leq \frac{f(x)}{f(y)} \leq c_{0} \mathrm{e}^{\varepsilon(x-y)} .
$$

By some standard arguments (see, e.g. Proposition 7 and Proposition 8 of Asmussen et al. (2003)), we can obtain the following results. 
Lemma 3. Let $f, f_{1}, \ldots, f_{k}$ be densities on $\mathbb{R}_{+}$such that $f_{i}(x) \sim c_{i} f(x)$ for $c_{i}>0, i=$ $1, \ldots, k$. If $f$ is a subexponential density then the following statements hold.

(a) For all $n_{1}, \ldots, n_{k} \in \mathbb{N}$,

$$
\left(f_{1}^{* n_{1}} * \cdots * f_{k}^{* n_{k}}\right)(x) \sim \sum_{i=1}^{k} n_{i} c_{i} f(x)
$$

and $f_{1}^{* n_{1}} * \cdots * f_{k}^{* n_{k}}$ is also a subexponential density.

(b) For any $\varepsilon>0$, these exist some $x_{\varepsilon} \geq 0$ and $0<N_{\varepsilon}<\infty$ such that

$$
\left(f_{1}^{* n_{1}} * \cdots * f_{k}^{* n_{k}}\right)(x) \leq N_{\varepsilon}(1+\varepsilon)^{n_{1}+\cdots+n_{k}} f(x)
$$

for all $x \geq x_{\varepsilon}$ and $n_{1}, \ldots, n_{k} \in \mathbb{N}$.

We will study the asymptotic behavior of $\boldsymbol{\Phi}(u)$ when $u$ is sufficiently large. It suffices to study the asymptotic behavior of $\boldsymbol{\Phi}_{+}(u)$. To this end, we need the following assumption.

Assumption 1. There exist a matrix $\boldsymbol{H}=\left[H_{i j}\right]_{i, j=1}^{m}$ and a distribution function $F$ with density $f$, such that, for $i, j=1, \ldots, m$,

$$
\lim _{x \rightarrow \infty} \frac{f_{i j}(x)}{f(x)}=H_{i j}<\infty, \quad H_{i j}>0 .
$$

Lemma 4. (a) For $\delta>0$, if Assumption 1 holds with $f \in \mathcal{L}_{d}$, we have

$$
\boldsymbol{g}_{\delta}(y) \sim \frac{1}{c} \boldsymbol{P}_{\delta}^{-1}\left[\boldsymbol{D}_{1} \circ \boldsymbol{f}(y)\right]
$$

(b) For $\delta=0$, if Assumption 1 holds with $\bar{F} \in \mathcal{L}_{d}$, we have

$$
\boldsymbol{g}_{0}(y) \sim \frac{1}{c} \boldsymbol{h} \boldsymbol{\pi}\left[\boldsymbol{D}_{1} \circ \overline{\boldsymbol{F}}(y)\right] .
$$

Proof. Recall Remark 1. By the Perron-Frobenius theorem we have

$$
\mathrm{e}^{-\boldsymbol{P}_{\delta} x}=\mathrm{e}^{-\rho_{\delta} x} \boldsymbol{h}_{\delta} \boldsymbol{\gamma}_{\delta}+O\left(\mathrm{e}^{-\beta x}\right),
$$

where $\beta>\rho_{\delta}$.

Firstly, for part (a), we have

$$
\frac{\boldsymbol{g}_{\delta}(y)}{f(y)}=\frac{1}{c} \int_{0}^{\infty} \mathrm{e}^{-\boldsymbol{P}_{\delta} x} \frac{\boldsymbol{D}_{1} \circ \boldsymbol{f}(x+y)}{f(x+y)} \frac{f(x+y)}{f(y)} \mathrm{d} x .
$$

By the dominated convergence theorem justified by (32), Assumption 1, and Lemma 2 with $\varepsilon<\rho_{\delta}$, we have

$$
\lim _{y \rightarrow \infty} \frac{\boldsymbol{g}_{\delta}(y)}{f(y)}=\frac{1}{c} \int_{0}^{\infty} \mathrm{e}^{-\boldsymbol{P}_{\delta} x} \lim _{y \rightarrow \infty} \frac{\boldsymbol{D}_{1} \circ \boldsymbol{f}(x+y)}{f(x+y)} \frac{f(x+y)}{f(y)} \mathrm{d} x=\frac{1}{c} \boldsymbol{P}_{\delta}^{-1}\left[\boldsymbol{D}_{1} \circ \boldsymbol{H}\right],
$$

which implies that (31) holds. 
Next, we consider part (b). By (32) we have

$$
\boldsymbol{g}_{0}(y)=\frac{1}{c} \boldsymbol{h} \boldsymbol{\pi}\left[\boldsymbol{D}_{1} \circ \overline{\boldsymbol{F}}(y)\right]+O\left(\frac{1}{c} \int_{0}^{\infty} \mathrm{e}^{-\beta x}\left[\boldsymbol{D}_{1} \circ \boldsymbol{f}(x+y)\right] \mathrm{d} x\right) .
$$

The condition $\bar{F} \in \mathcal{L}_{d}$ also implies that $f \in \mathcal{L}_{d}$. It follows from Lemma 4.4(1) of Tang and Wei (2010) that $f(x) \sim o(\bar{F}(x))$. Then, by Assumption 1 and the dominated convergence theorem again, it is not hard to see that, compared with $\boldsymbol{h} \boldsymbol{\pi}\left[\boldsymbol{D}_{1} \circ \overline{\boldsymbol{F}}(y)\right]$, the matrix-valued function

$$
\int_{0}^{\infty} \mathrm{e}^{-\beta x}\left[\boldsymbol{D}_{1} \circ \boldsymbol{f}(x+y)\right] \mathrm{d} x
$$

is asymptotically negligible. This completes the proof.

To continue, we need to specify the matrix $\boldsymbol{I}-\boldsymbol{G}_{\delta}$, where $\boldsymbol{G}_{\delta}=\int_{0}^{\infty} \boldsymbol{g}_{\delta}(y) \mathrm{d} y$. Setting $s=0$ in (22) gives

$$
\boldsymbol{P}_{\delta}\left[\boldsymbol{I}-\boldsymbol{G}_{\delta}\right]=\frac{1}{c}[\delta \boldsymbol{I}-\boldsymbol{D}] .
$$

For $\delta>0, \boldsymbol{P}_{\delta}$ is nonsingular. Then (33) gives

$$
\boldsymbol{I}-\boldsymbol{G}_{\delta}=\frac{1}{c} \boldsymbol{P}_{\delta}^{-1}[\delta \boldsymbol{I}-\boldsymbol{D}], \quad \delta>0 .
$$

For $\delta=0$, firstly, we have

$$
\begin{aligned}
\boldsymbol{h} \boldsymbol{\pi}\left[\boldsymbol{I}-\boldsymbol{G}_{0}\right] & =\boldsymbol{h} \boldsymbol{\pi}-\frac{1}{c} \int_{0}^{\infty} \int_{0}^{\infty} \boldsymbol{h} \boldsymbol{\pi} \mathrm{e}^{-\boldsymbol{P}_{0} x}\left[\boldsymbol{D}_{1} \circ \boldsymbol{f}(x+y)\right] \mathrm{d} x \mathrm{~d} y \\
& =\boldsymbol{h} \boldsymbol{\pi}\left[\boldsymbol{I}-\frac{1}{c} \boldsymbol{D}_{1} \circ \boldsymbol{\mu}\right]
\end{aligned}
$$

thanks to $\boldsymbol{\pi} \boldsymbol{P}_{0}=\mathbf{0}$, where $\boldsymbol{\mu}=\left[\mu_{i j}\right]_{i, j=1}^{m}$. Then, by (34) and (33) with $\delta=0$, we obtain

$$
\left[\boldsymbol{h} \boldsymbol{\pi}-\boldsymbol{P}_{0}\right]\left[\boldsymbol{I}-\boldsymbol{G}_{0}\right]=\boldsymbol{h} \boldsymbol{\pi}\left[\boldsymbol{I}-\frac{1}{c} \boldsymbol{D}_{1} \circ \boldsymbol{\mu}\right]+\frac{1}{c} \boldsymbol{D} .
$$

The matrix $\boldsymbol{h} \boldsymbol{\pi}-\boldsymbol{P}_{0}$ is nonsingular and $\left[\boldsymbol{h} \boldsymbol{\pi}-\boldsymbol{P}_{0}\right] \boldsymbol{h} \boldsymbol{\pi}=\boldsymbol{h} \boldsymbol{\pi}$. Then (35) gives

$$
\boldsymbol{I}-\boldsymbol{G}_{0}=\boldsymbol{h} \boldsymbol{\pi}\left[\boldsymbol{I}-\frac{1}{c} \boldsymbol{D}_{1} \circ \boldsymbol{\mu}\right]+\frac{1}{c}\left[\boldsymbol{h} \boldsymbol{\pi}-\boldsymbol{P}_{0}\right]^{-1} \boldsymbol{D} .
$$

Lemma 5. (a) For $\delta>0$, if Assumption 1 holds with $f \in \varsigma_{d}$, we have

$$
\boldsymbol{\Pi}_{\delta}(y) \sim \frac{1}{c}\left[\boldsymbol{I}-\boldsymbol{G}_{\delta}\right]^{-1} \boldsymbol{P}_{\delta}^{-1}\left[\boldsymbol{D}_{1} \circ \boldsymbol{f}(y)\right]\left[\boldsymbol{I}-\boldsymbol{G}_{\delta}\right]^{-1} .
$$

(b) For $\delta=0$, if Assumption 1 holds with $\bar{F} \in \varsigma_{d}$, we have

$$
\boldsymbol{\Pi}_{0}(y) \sim \frac{1}{c}\left[\boldsymbol{I}-\boldsymbol{G}_{0}\right]^{-1} \boldsymbol{h} \boldsymbol{\pi}\left[\boldsymbol{D}_{1} \circ \overline{\boldsymbol{F}}(y)\right]\left[\boldsymbol{I}-\boldsymbol{G}_{0}\right]^{-1} .
$$


Proof. We only prove (a) since (b) can be obtained similarly. Let $\check{\boldsymbol{g}}_{\delta}(y)=\left[\check{g}_{\delta, i j}(y)\right]_{i, j=1}^{m}$, where $\check{g}_{\delta, i j}(y)=g_{\delta, i j}(y) /\left[\boldsymbol{G}_{\delta}\right]_{i j}$. By (31) we have

$$
\check{g}_{\delta, i j}(y) \sim \frac{\left[\boldsymbol{P}_{\delta}^{-1}\left[\boldsymbol{D}_{1} \circ \boldsymbol{H}\right]\right]_{i j}}{c\left[\boldsymbol{G}_{\delta}\right]_{i j}} f(y) .
$$

For fixed $n$, we have

$$
\begin{aligned}
{\left[\boldsymbol{g}_{\delta}^{* n}(y)\right]_{i j} } & =\sum_{i_{1}=1}^{m} \cdots \sum_{i_{n-1}=1}^{m}\left(g_{\delta, i i_{1}} * \cdots * g_{\delta, i_{n-1} j}\right)(y) \\
& =\sum_{i_{1}=1}^{m} \cdots \sum_{i_{n-1}=1}^{m}\left(\left[\boldsymbol{G}_{\delta}\right]_{i i_{1}} \cdots\left[\boldsymbol{G}_{\delta}\right]_{i_{n-1} j}\right)\left(\check{g}_{\delta, i i_{1}} * \cdots * \check{g}_{\delta, i_{n-1} j}\right)(y) .
\end{aligned}
$$

By Lemma 3(a) and (37), we have

$$
\begin{aligned}
{\left[\boldsymbol{g}_{\delta}^{* n}(y)\right]_{i j} \sim \sum_{i_{1}=1}^{m} \cdots \sum_{i_{n-1}=1}^{m} } & \left(\left[\boldsymbol{G}_{\delta}\right]_{i i_{1}} \cdots\left[\boldsymbol{G}_{\delta}\right]_{i_{n-1} j}\right) \\
& \times\left(\frac{\left[\boldsymbol{P}_{\delta}^{-1}\left[\boldsymbol{D}_{1} \circ \boldsymbol{H}\right]\right]_{i i_{1}}}{c\left[\boldsymbol{G}_{\delta}\right]_{i i_{1}}}+\cdots+\frac{\left[\boldsymbol{P}_{\delta}^{-1}\left[\boldsymbol{D}_{1} \circ \boldsymbol{H}\right]\right]_{i_{n-1} j}}{c\left[\boldsymbol{G}_{\delta}\right]_{i_{n-1} j}}\right) f(y) .
\end{aligned}
$$

In matrix form, we have

$$
\begin{aligned}
\boldsymbol{g}_{\delta}^{* n}(y) & \sim \frac{1}{c} \sum_{k=0}^{n-1} \boldsymbol{G}_{\delta}^{k} \boldsymbol{P}_{\delta}^{-1}\left[\boldsymbol{D}_{1} \circ \boldsymbol{H}\right] \boldsymbol{G}_{\delta}^{n-1-k} f(y) \\
& \sim \frac{1}{c} \sum_{k=0}^{n-1} \boldsymbol{G}_{\delta}^{k} \boldsymbol{P}_{\delta}^{-1}\left[\boldsymbol{D}_{1} \circ \boldsymbol{f}(\boldsymbol{y})\right] \boldsymbol{G}_{\delta}^{n-1-k}
\end{aligned}
$$

By Lemma 3(b) and (38), we know that, for any $\varepsilon>0$, there exists some $N_{\varepsilon}<\infty$ such that, for large $y$,

$$
\left[\boldsymbol{g}_{\delta}^{* n}(y)\right]_{i j} \leq \sum_{i_{1}=1}^{m} \cdots \sum_{i_{n-1}=1}^{m}\left(\left[\boldsymbol{G}_{\delta}\right]_{i i_{1}} \cdots\left[\boldsymbol{G}_{\delta}\right]_{i_{n-1} j}\right) N_{\varepsilon}(1+\varepsilon)^{n} f(y),
$$

that is,

$$
\boldsymbol{g}_{\delta}^{* n}(y) \leq \boldsymbol{G}_{\delta}^{n} N_{\varepsilon}(1+\varepsilon)^{n} f(y) .
$$

Since $\boldsymbol{G}_{\delta}$ is strictly substochastic, we can choose $\varepsilon$ small enough such that the spectral radius of $(1+\varepsilon) \boldsymbol{G}_{\delta}$ is less than 1 . Then, by the dominated convergence theorem and (39), we obtain $\boldsymbol{\Pi}_{\delta}(y) \sim \frac{1}{c} \sum_{n=1}^{\infty} \sum_{k=0}^{n-1} \boldsymbol{G}_{\delta}^{k} \boldsymbol{P}_{\delta}^{-1}\left[\boldsymbol{D}_{1} \circ \boldsymbol{f}(y)\right] \boldsymbol{G}_{\delta}^{n-1-k}=\frac{1}{c}\left[\boldsymbol{I}-\boldsymbol{G}_{\delta}\right]^{-1}\left[\boldsymbol{P}_{\delta}^{-1}\left[\boldsymbol{D}_{1} \circ \boldsymbol{f}(y)\right]\right]\left[\boldsymbol{I}-\boldsymbol{G}_{\delta}\right]^{-1}$.

This completes the proof.

Let $\bar{\Omega}_{i j}(u)=\int_{u}^{\infty} \omega_{i j}(x) \mathrm{d} x$ and $\bar{F}_{e, i j}(u)=\int_{u}^{\infty} \bar{F}_{i j}(x) \mathrm{d} x$, and set $\overline{\boldsymbol{\Omega}}(u)=\left[\bar{\Omega}_{i j}(u)\right]_{i, j=1}^{m}$ and $\overline{\boldsymbol{F}}_{e}(u)=\left[\bar{F}_{e, i j}(u)\right]_{i, j=1}^{m}$. 
Theorem 3. (a) For $\delta>0$, assume that $\lim _{u \rightarrow \infty} \omega_{i j}(u) / \bar{F}_{i j}(u)=\kappa>0$ and that Assumption 1 holds with $f \in S_{d}$. Then

$$
\boldsymbol{\Phi}_{+}(u) \sim \frac{1}{c}\left[\boldsymbol{I}-\boldsymbol{G}_{\delta}\right]^{-1} \boldsymbol{P}_{\delta}^{-1}\left[\boldsymbol{D}_{1} \circ \boldsymbol{\omega}(u)\right] .
$$

(b) For $\delta=0$, assume that $\lim _{u \rightarrow \infty} \bar{\Omega}_{i j}(u) / \bar{F}_{e, i j}(u)=\kappa>0$ and that Assumption 1 holds with $\bar{F} \in \varsigma_{d}$. Then

$$
\boldsymbol{\Phi}_{+}(u) \sim \frac{1}{c}\left[\boldsymbol{I}-\boldsymbol{G}_{0}\right]^{-1} \boldsymbol{h} \boldsymbol{\pi}\left[\boldsymbol{D}_{1} \circ \overline{\boldsymbol{\Omega}}(u)\right] .
$$

Proof. We only show part (a) since part (b) can be obtained similarly. First, note that

$$
\begin{aligned}
\boldsymbol{Z}_{\delta}(u)= & \frac{1}{c} \int_{0}^{\infty} \int_{-c / r}^{0} \mathrm{e}^{-\boldsymbol{P}_{\delta} x}\left[\boldsymbol{D}_{1} \circ \boldsymbol{f}(x+u-y)\right] \boldsymbol{\Phi}_{-}(y) \mathrm{d} y \mathrm{~d} x \\
& +\frac{1}{c} \int_{0}^{\infty} \mathrm{e}^{-\boldsymbol{P}_{\delta} x}\left[\boldsymbol{D}_{1} \circ \boldsymbol{\omega}(x+u)\right] \mathrm{d} x \\
:= & \boldsymbol{M}_{1}(u)+\boldsymbol{M}_{2}(u) .
\end{aligned}
$$

The condition $f \in \varsigma_{d}$ implies that $F \in \varsigma_{\text {and }} \bar{F} \in \mathcal{L}_{d}$. We have

$$
\lim _{x \rightarrow \infty} \frac{\omega_{i j}(x)}{\bar{F}(x)}=\lim _{x \rightarrow \infty} \frac{\omega_{i j}(x)}{\bar{F}_{i j}(x)} \frac{\bar{F}_{i j}(x)}{\bar{F}(x)}=\kappa H_{i j}
$$

thanks to Assumption 1 and l'Hôpital's rule, which implies that $\omega_{i j} \in \mathcal{L}_{d}$, since the class $\mathscr{L}_{d}$ is closed with respect to asymptotic equivalence. Thus, by exactly the same arguments as in the proof of Lemma 4(a), we can show that all entries of $\boldsymbol{M}_{2}(u)$ belong to the class $\delta_{d}$ and

$$
\boldsymbol{M}_{2}(u) \sim \frac{\kappa}{c} \boldsymbol{P}_{\delta}^{-1}\left[\boldsymbol{D}_{1} \circ \boldsymbol{H}\right] \bar{F}(u) .
$$

The condition $f \in \wp_{d}$ implies that $f(x) \sim o(\bar{F}(x))$ due to Lemma 4.4(1) of Tang and Wei (2010) again. Then, by Assumption 1 and the dominated convergence theorem, we know that

$$
\frac{\boldsymbol{M}_{1}(u)}{\bar{F}(u)}=\frac{1}{c} \int_{0}^{\infty} \int_{-c / r}^{0} \mathrm{e}^{-\boldsymbol{P}_{\delta} x} \frac{\boldsymbol{D}_{1} \circ \boldsymbol{f}(x+u-y)}{f(x+u-y)} \frac{f(x+u-y)}{\bar{F}(u)} \boldsymbol{\Phi}_{-}(y) \mathrm{d} y \mathrm{~d} x
$$

tends to a zero matrix as $u \rightarrow \infty$. By this and (40), we have $\boldsymbol{M}_{1}(u) \sim o\left(\boldsymbol{M}_{2}(u)\right)$, and

$$
\boldsymbol{Z}_{\delta}(u) \sim \frac{\kappa}{c} \boldsymbol{P}_{\delta}^{-1}\left[\boldsymbol{D}_{1} \circ \boldsymbol{H}\right] \bar{F}(u) .
$$

It is more convenient to write (41) in the form

$$
Z_{\delta, i j}(u) \sim a_{i j} \bar{F}(u),
$$

where $Z_{\delta, i j}(u)$ is the $(i, j)$ th entry of $\boldsymbol{Z}_{\delta}(u)$ and

$$
a_{i j}=\frac{\kappa}{c}\left[\boldsymbol{P}_{\delta}^{-1}\left[\boldsymbol{D}_{1} \circ \boldsymbol{H}\right]\right]_{i j} .
$$

Similarly, we write (36) in the form

$$
\Pi_{\delta, i j}(y) \sim b_{i j} f(y),
$$


where $\Pi_{\delta, i j}(y)$ is the $(i, j)$ th entry of $\Pi_{\delta}(y)$ and

$$
b_{i j}=\frac{1}{c}\left[\left[\boldsymbol{I}-\boldsymbol{G}_{\delta}\right]^{-1} \boldsymbol{P}_{\delta}^{-1}\left[\boldsymbol{D}_{1} \circ \boldsymbol{H}\right]\left[\boldsymbol{I}-\boldsymbol{G}_{\delta}\right]^{-1}\right]_{i j} .
$$

For any fixed $x_{0}>0$ and $u>2 x_{0}$, by (30), we have

$$
\begin{aligned}
\Phi_{+, i j}(u) & =Z_{\delta, i j}(u)+\sum_{k=1}^{m} \int_{0}^{u} \Pi_{\delta, i k}(y) Z_{\delta, k j}(u-y) \mathrm{d} y \\
& =Z_{\delta, i j}(u)+\sum_{k=1}^{m}\left(\int_{0}^{x_{0}}+\int_{x_{0}}^{u-x_{0}}+\int_{u-x_{0}}^{u}\right) \Pi_{\delta, i k}(y) Z_{\delta, k j}(u-y) \mathrm{d} y \\
& :=Z_{\delta, i j}(u)+\sum_{k=1}^{m}\left(L_{i k j, 1}(u)+L_{i k j, 2}(u)+L_{i k j, 3}(u)\right) .
\end{aligned}
$$

By $\bar{F} \in \mathscr{L}_{d}$, (42), and the dominated convergence theorem,

$$
L_{i k j, 1}(u) \sim \int_{0}^{x_{0}} \Pi_{\delta, i k}(y) \mathrm{d} y Z_{\delta, k j}(u) .
$$

By $F \in 8$, (42), and (43), it is not hard to see that (see, e.g. Klùppelberg (1988))

$$
\limsup _{x_{0} \rightarrow \infty} \limsup _{u \rightarrow \infty} \frac{L_{i k j, 2}(u)}{\bar{F}(u)}=\limsup _{x_{0} \rightarrow \infty} \limsup _{u \rightarrow \infty} b_{i k} a_{k j} \int_{x_{0}}^{u-x_{0}} \frac{\bar{F}(u-y)}{\bar{F}(u)} \mathrm{d} F(y)=0 .
$$

Recall that the penalty function is bounded. Furthermore, $Z_{\delta, k j}(u)$ must be bounded by some constant, say $d>0$. Then

$$
L_{i k j, 3}(u) \leq d \int_{u-x_{0}}^{u} \Pi_{\delta, i k}(y) \mathrm{d} y \sim \mathrm{d} b_{i k}\left[\bar{F}\left(u-x_{0}\right)-\bar{F}(u)\right] \sim o(\bar{F}(u)) .
$$

Thus, letting first $u \rightarrow \infty$ and then $x_{0} \rightarrow \infty$, using (42) and (45)-(47), we obtain

$$
\int_{0}^{u} \Pi_{\delta, i k}(y) Z_{\delta, k j}(u-y) \mathrm{d} y \sim \int_{0}^{\infty} \Pi_{\delta, i k}(y) \mathrm{d} y a_{k j} \bar{F}(u),
$$

which together with (42) and (44) gives

$$
\Phi_{+, i j}(u) \sim a_{i j} \bar{F}(u)+\sum_{k=1}^{m} \int_{0}^{\infty} \Pi_{\delta, i k}(y) \mathrm{d} y a_{k j} \bar{F}(u) .
$$

Rewriting the above equation in matrix forms gives

$$
\begin{aligned}
\boldsymbol{\Phi}_{+}(u) & \sim \frac{\kappa}{c} \boldsymbol{P}_{\delta}^{-1}\left[\boldsymbol{D}_{1} \circ \boldsymbol{H}\right] \bar{F}(u)+\frac{\kappa}{c} \int_{0}^{\infty} \boldsymbol{\Pi}_{\delta}(y) \mathrm{d} y \boldsymbol{P}_{\delta}^{-1}\left[\boldsymbol{D}_{1} \circ \boldsymbol{H}\right] \bar{F}(u) \\
& =\frac{\kappa}{c}\left[\boldsymbol{I}-\boldsymbol{G}_{\delta}\right]^{-1} \boldsymbol{P}_{\delta}^{-1}\left[\boldsymbol{D}_{1} \circ \boldsymbol{H}\right] \bar{F}(u) \\
& \sim \frac{1}{c}\left[\boldsymbol{I}-\boldsymbol{G}_{\delta}\right]^{-1} \boldsymbol{P}_{\delta}^{-1}\left[\boldsymbol{D}_{1} \circ \boldsymbol{\omega}(u)\right] .
\end{aligned}
$$

This completes the proof. 
Remark 3. Theorem 3 generalizes the corresponding results in Cai (2007) and Yin and Wang (2010). As remarked in Yin and Wang (2010), the assumption on the penalty function is not very restrictive. In fact, asymptotic behaviors of several interesting ruin related functions, such as the Laplace transform of the time to absolute ruin, the absolute ruin probability, and the (discounted) distribution of the deficit at ruin, can be obtained from Theorem 3 .

\section{Acknowledgements}

The authors would like to thank the anonymous referee for constructive comments that improved the contents and presentation of this paper. This research was funded by the Chongqing University Postgraduates' Science and Innovation Fund (project number 200911B1B0110327), and the Research Grants Council of the Hong Kong Special Administrative Region, China (project number HKU 754008H).

\section{References}

Ahn, S. AND BAdEscu, A. L. (2007). On the analysis of the Gerber-Shiu discounted penalty function for risk processes with Markovian arrivals. Insurance Math. Econom. 41, 234-249.

Asmussen, S. (2000). Ruin Probabilities. World Scientific, River Edge, NJ.

Asmussen, S. (2003). Applied Probability and Queues, 2nd edn. Springer, New York.

Asmussen, S., Foss, S. And Korshunov, D. (2003). Asymptotics for sums of random variables with local subexponential behavior. J. Theoret. Prob. 16, 489-518.

Asmussen, S., Fløe Henriksen, L. And Klüppelberg, C. (1994). Large claims approximations for risk processes in a Markovian environment. Stoch. Process. Appl. 54, 29-43.

Breuer, L. (2008). First passage times for Markov additive processes with positive jumps of phase type. J. Appl. Prob. 45, 779-799.

BADESCU A. L. et al. (2005a). The joint density of the surplus prior to ruin and the deficit at ruin for a correlated risk process. Scand. Actuarial J. 2005, 433-446.

BADESCU A. L. et al. (2005b). Risk processes analyzed as fluid queues. Scand. Actuarial J. 2005, 127-141.

CAI, J. (2007). On the time value of absolute ruin with debit interest. Adv. Appl. Prob. 39, 343-359.

Cheung, E. C. K. AND Landriault, D. (2010). A generalized penalty function with the maximum surplus prior to ruin in a MAP risk model. Insurance Math. Econom. 46, 127-134.

Dassios, A. And Embrechts, P. (1989). Martingales and insurance risk. Commun. Statist. Stoch. Models 5, 181-217.

Embrechts, P. AND Schmidl, H. (1994). Ruin estimation for a general insurance model. Adv. Appl. Prob. 26, 404-422.

Embrechts, P., Klüppelberg, C. And Mikosch, T. (1997). Modelling Extremal Events. Springer, Berlin.

Gerber, H. U. 1971. Der Einfluss von Zins auf die Ruinwahrscheinlichkeit. Bull. Swiss Assoc. Actuaries 71, 63-70.

Gerber, H. U. And Shiu, E. S. W. (1998). On the time value of ruin. N. Amer. Actuarial J. 2, 48-78.

Gerber, H. U. AND YANG, H. (2007). Absolute ruin probabilities in a jump diffusion risk model with investment. N. Amer. Actuarial J. 11, 159-169.

KLÜPPElberg, C. (1988). Subexponential distributions and integrated tails. J. Appl. Prob. 25, 132-141.

KlÜPPELBERG, C. (1989). Subexponential distributions and characterizations of related classes. Prob. Theory Relat. Fields 82, 259-269.

Konstantinides, D. G., NG, K. W. And TAng, Q. (2010). The probabilities of absolute ruin in the renewal risk model with constant force of interest. J. Appl. Prob. 47, 323-334.

LinZ, P. (1985). Analytical and Numerical Methods for Volterra Equations (SIAM Stud. Appl. Math. 7). Society for Industrial and Applied Mathematics, Philadelphia, PA.

Rolski, T., Schmidli, H., Schmidt, V. ANd Teugels, J. (1999). Stochastic Processes for Insurance and Finance. John Wiley, Chichester.

TANG, Q. AND WeI, L. (2010). Asymptotic aspects of the Gerber-Shiu function in the renewal risk model using Wiener-Hopf factorization and convolution equivalence. Insurance Math. Econom. 46, 19-31.

YIN, C. AND WANG, C. (2010). The perturbed compound Poisson risk process with investment and debit interest. Methodology Comput. Appl. Prob. 12, 391-413. 